\title{
Adipocyte-myofibroblast transition: linking intradermal fat loss to skin fibrosis in SSc
}

$\mathrm{M}$ yofibroblasts are considered the primary fibrogenic effector cells in systemic sclerosis (SSc), but the origin of these cells within fibrotic lesions is a matter of debate. A new study published in Arthritis \& Rheumatology shows that the majority of dermal myofibroblasts in fibrotic skin arise from adiponectin-positive progenitors resident in the skin, and suggest that a differentiation process the investigators term adipocyte-myofibroblast transition (AMT) could have an important role in the pathogenesis of skin fibrosis.

Substantial loss of the intradermal adipose layer is a consistent hallmark of SSc and animal models of experimentally induced fibrosis, but little is known about its progression and consequences. "We speculated that intradermal adipocytes change their cell fates by transforming into scar-forming myofibroblasts," explains John Varga, co-author of the study. "This seemed a reasonable hypothesis, since we already knew from in vitro studies that adipocytes can be coaxed to become myofibroblasts under the influence of transforming growth factor $\beta$ (TGF- $\beta$ ) and Wnt, signalling proteins that are implicated in pathogenesis of SSc."

To investigate the AMT hypothesis, the research team led by Roberta Gonçalves Marangoni first examined the time-course for the development of bleomycin-induced fibrosis, a well-established mouse model of dermal fibrosis. In C57BL/6J mice given daily subcutaneous injections of bleomycin, morphological and gene expression analysis revealed that marked depletion of the intradermal adipose layer preceded the development of dermal fibrosis. By day 5 of the bleomycin injections, more than half of the adipose layer was lost, and over the first 2 weeks, adipocytes in the intradermal layer progressively decreased in both size and number. By contrast, the dermis was not substantialy thickened until day 14. An increase in the expression of fibrogenic genes in the lesional skin likewise followed a decline in the expression of adipogenic genes. "[These findings] indicated that loss of intradermal adipose tissue in the scleroderma model is a primary event in fibrosis, rather than a consequence of it," says Varga.

An early and transient increase in the number of apoptotic adipocytes was observed but could not account for the extent of intradermal fat loss, so the investigators performed lineagetracing studies to identify the fate of intradermal adipocytes. Marangoni's team generated transgenic mice in which mature adipocytes were permanently marked by the tandem dimer tomato (tdT) fluorophore.

Experimental fibrosis was induced in these transgenic mice by bleomycin injections; after 2-3 weeks, the dermis was thickened and accumulation of collagen was evident. "We were able to show that the fibrotic skin in these transgenic mice was full of myofibroblasts that were tdT-positive, indicating that these cells originated from adipocytes," recounts Varga. "Other cells in the skin, such as inflammatory cells, did not originate from adipocytes."

In the transgenic mice, by day 5 of bleomycin injections, the intradermal adipose layer was substantially attenuated, but no tdT-positive cells were found in the dermis. By day 8, cells were detected in the intradermal fat that expressed tdT and the adipocyte-specific marker perilipin as well as the myofibroblast marker a-smooth muscle actin (SMA). By day 14 and the final bleomycin injection, multiple cells in the lower dermis expressed tdT and a-SMA, but no longer expressed perilipin. In skin harvested at day 21, most of the myofibroblasts in the fibrotic dermis were tdT-positive.
Adipocyte cell-fate transition was then further characterized ex vivo in adiposederived cells. Incubation of human subcutaneous adipocytes with TGF- $\beta$ resulted in time-dependent changes in cell morphology and biochemistry so that the cells lost adipocytic characteristics and became fibroblast-like.

"At intermediate time points we detected 'transitional cells'-that is, cells that retained adipocyte characteristics but had already acquired features of myofibroblasts," says Varga. "At later time points, mostly myofibroblasts were found."

Genome-wide expression profiling of the cells confirmed the transcriptional reprogramming of the adipocytes: treatment with TGF- $\beta$ led to increased expression of a number of fibrogenic genes and suppression of multiple adipogenic genes in the adipocyte cultures.

Varga highlights that the results have implications for understanding and treating scleroderma, and possibly also for other fibrotic diseases such as liver cirrhosis, bone marrow fibrosis and even cancer.

The researchers plan to examine AMT in other mouse models of fibrosis and in skin from patients with SSc, to further characterize the process and define the molecular signals that regulate it. "Most importantly," he concludes, "we are exploring ways to therapeutically manipulate the AMT process in order to attenuate fibrosis in SSc."

Sarah Onuora

\footnotetext{
Original article Marangoni, R. G. et al. Myofibroblasts in cutaneous fibrosis originate from adiponectin-positive intradermal progenitors. Arthritis Rheumatol. doi:10.1002/ art.38990
} 